\section{'A bridge too far' - the negative impact of a bridge prosthesis on gingival health and its conservative management}

\author{
A. Gopakumar*1 and E. L. Boyle ${ }^{2}$
}

\section{VERIFIABLE CPD PAPER}

IN BRIEF

- Highlights the alternative non-surgical and conservative management of inflamed gingivae adjacent to a failing bridge.

- Stresses the importance of careful planning of bridge and pontic design, as well as the use of good clinical technique.

- Emphasises the need for access for effective plaque removal in maintaining harmony with the adjacent periodontium.

Bridges are commonly used to replace missing teeth. Periodontal health may, however, be compromised by ill-fitting margins and excess cement. This case report describes the conservative management of chronic gingival inflammation adjacent to the resin-bonded retainers of an anterior bridge by gingival remodelling via a removable prosthesis. This avoided the need for surgical intervention and re-established a suitable gingival profile before placement of a definitive cantilever resin-bonded bridge.

\section{CASE REPORT}

A 25-year-old female was referred to the Liverpool University Dental Hospital by her general dental practitioner, who was concerned about the gingival inflammation adjacent to the patient's anterior bridge. The referral letter stated that the patient required a gingivectomy localised to the 11 and 12 bridge abutments which supported a three-unit resin-bonded bridge, replacing the 11 .

The patient reported that she was unhappy with the aesthetics of the 11 pontic, as it did not match the appearance of the adjacent teeth. She was also concerned about the redness that had developed on the gingivae and found plaque removal around the bridge and adjacent teeth difficult. The current fixed-fixed resin-bonded bridge replacing the 11 was fitted three months ago and had already debonded twice within that period (Figs 1a-b).

The patient was asthmatic and an exsmoker. Clinical examination revealed gingivitis adjacent to the bridge retainers and pontic on the buccal and palatal

\footnotetext{
Specialist Registrar in Restorative Dentistry; ${ }^{2}$ Senior Lecturer/Honorary Consultant in Restorative Dentistry and Assistant Head of Liverpool Dental School, Liverpool University Dental Hospital, Pembroke Place, Liverpool, L3 5PS

*Correspondence to: Miss Ashish Gopakumar Email: ashish_gopakumar@yahoo.co.uk
}

\section{Refereed Paper}

Accepted 10 May 2013

DOI: 10.1038/sj.bdj.2013.877

${ }^{\circ}$ British Dental Journal 2013; 215: 273-276 aspects. Plaque deposits were also present especially below the fitting surface of the bridge pontic. On probing, there was residual cement subgingivally adjacent to the retainers. A periapical radiograph showed no apical pathology or bone loss associated with the 21 . The occlusion was favourable as she had an increased overjet and decreased overbite, hence avoiding any contact on the bridge in the intercuspal position or excursive movements. A diagnosis of chronic marginal gingivitis associated with an unaesthetic and poorly designed bridge was made.

\section{TREATMENT}

The management options were discussed with the patient which included removal of the existing bridge followed by either electrosurgery procedure or a conservative non-surgical approach of using a removable prosthesis to contour and remodel the gingivae while also providing non-surgical periodontal therapy and improving the patient's oral hygiene status.

The non-surgical approach was agreed upon and the patient was warned that at each appointment the removable prosthesis would not seat fully due to incremental additions of resin material on the fitting surface. She was informed that over the course of days/weeks the prosthesis would seat further. Impressions for study models and a working cast were taken. The area on the working model which corresponded to the hyperplastic gingivae was reduced in

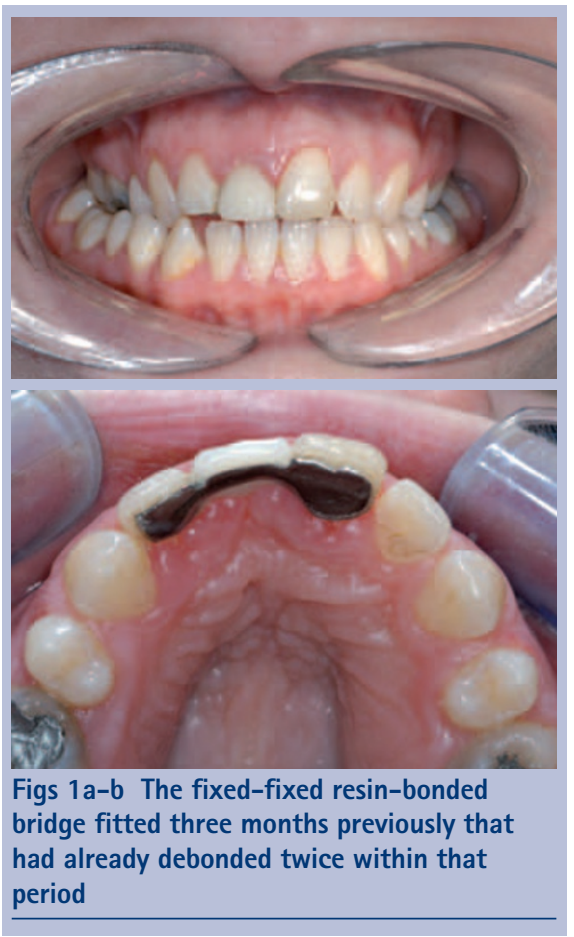

height by 1-1.5 mm using a fissure bur. A removable prosthesis, replacing the 11 , was fabricated using a clear light-cured resin (Eclipse, Denstply, UK). The bridge was sectioned and removed, following which deposits of residual cement and calculus were eliminated via ultrasonic scaling (Figs 2a-b).

At the same appointment the patient was given oral hygiene instructions and use of interdental cleaning aids was demonstrated. She was fitted with the removable prosthesis replacing the 11 , and was advised to remove it at night. As expected, the prosthesis did 
not seat fully. However, her occlusion was very favourable and there was no premature contact or alteration in the vertical dimension with the prosthesis in situ.

The patient was seen initially at intervals of two weeks and this was then subsequently extended to monthly over a six-month period. The patient's gingival health continuously improved as she was meticulous with her oral hygiene regime. At each visit the gingival contours and profile were examined. The inflammation had subsided and the gingivae showed reduction in height. Incremental additions of the light-cured resin (Eclipse, Dentsply, UK) were made to selected areas of the fitting surface of the removable prosthesis, which corresponded to the gingival hyperplasia (Fig. 3). This repeated application of pressure reduced the bulkiness of the gingivae and contoured it to a suitable profile.

Once a satisfactory gingival profile was achieved, options for definitive replacement of the 21 were discussed with the patient. As previously, the patient was keen to have a fixed prosthesis. A single tooth implant was not feasible due to financial constraints. Following agreement, the patient was provided with a twounit cantilever resin-bonded bridge using the 21 as a bridge abutment (Figs 4a-b).

This was fitted using a self-curing adhesive resin cement (Panavia 21, Kuraray Dental, UK) and use of interdental cleaning aids such as superfloss was demonstrated to the patient so as to enable her to maintain optimal plaque control. The patient was pleased with the improved aesthetics. She was subsequently reviewed and continued to maintain good gingival health (Figs 5a-b).

\section{DISCUSSION}

A healthy periodontium is a prerequisite for a successful outcome of a fixed prosthesis. ${ }^{1}$ Careful consideration needs to be given to factors such as bridge design, number/type/preparation of abutment teeth, pontic design and cementation. The location, contours and emergence profile of the retainer margins will determine the response of the gingival tissues to the prosthesis. Harmony between the prosthesis and the periodontium is critical. If this balance is not achieved it can result in a compromised periodontium and suboptimal aesthetics. Patient factors such as a failure to comply with oral hygiene instructions almost certainly will compromise a successful outcome.

The bridge prosthesis can have a direct negative impact on the health of the adjacent periodontium. Interestingly, the three most common complications associated with resin-bonded prosthesis are prosthesis debonding, tooth discoloration and caries. ${ }^{2}$ Periodontal disease was reported as the fifth common complication, just after porcelain fracture.

Conversely, there is no evidence to support that periodontal factors will have any direct effect on the survival of a bridge.

The biologic width was not compromised in this case by the restoration margin. The concept of the biologic width was introduced by Gargiulo et al. in $1961^{3}$ and it incorporated the height of the junctional epithelium and the connective tissue attachment. This resulted in a total height of $2.04 \mathrm{~mm}$, based on histological measurements (Fig. 6). It has been recommended that there should be between $3 \mathrm{~mm}$ and $5.25 \mathrm{~mm}$ of supracrestal tissue between the bone and the margin of the restoration. ${ }^{1,4,5}$

A removable prosthesis was made where resin material could be incrementally added to in localised areas which corresponded to the hyperplastic tissues in the mouth. This application of constant pressure achieved gingival contouring and tissue remodelling. This was a simple, quick and easy technique to use without the need for any bonding agent. However, use of this light-cured resin (Eclipse, Dentsply, UK) in this case required a specific processing unit from the manufacturer. A similar prosthesis can be made using methylmethacrylate acrylic and increments can be built up using chairside acrylic materials, where this may be unavailable or the dental laboratory is off-site.

Case selection is crucial to ensure that the optimal treatment is delivered successfully to ensure a satisfactory clinical outcome as well as a high level of patient satisfaction. Treatment options for missing teeth have been discussed extensively in the literature. Success rates for resin-bonded bridges have improved over the years owing to advancements in cementation materials and micromechanical bonding. Studies have quoted success rates of $94 \%$ after three years ${ }^{6}$ and 94\% after nearly three years. ${ }^{7}$ A recent systematic review found a five-year survival
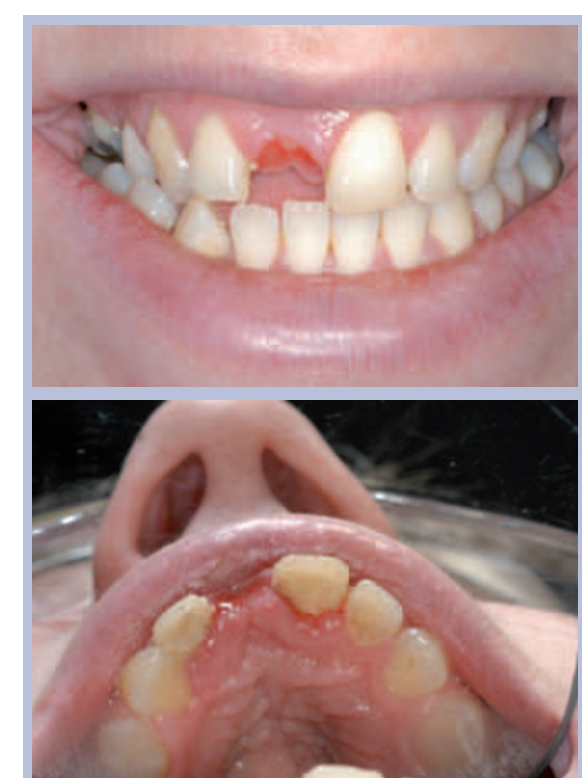

Figs $2 a-b$ The bridge was sectioned and removed, following which deposits of residual cement and calculus were removed with ultrasonic scaling

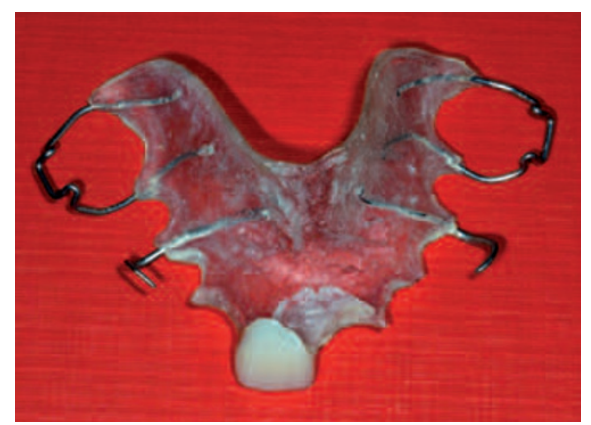

Fig. 3 Incremental additions of the light-cured resin were made to selected areas of the fitting surface of the removable prosthesis, which corresponded to the gingival hyperplasia

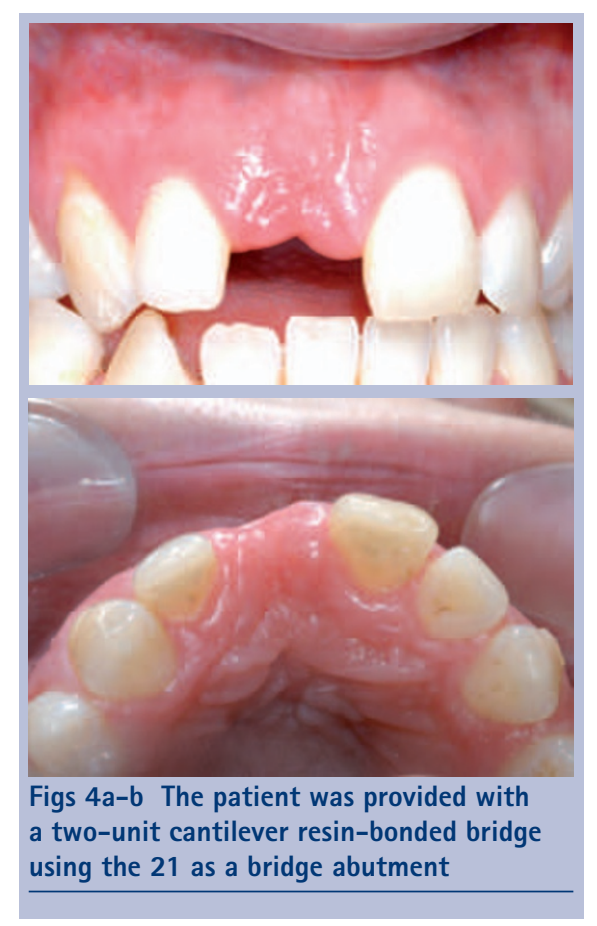




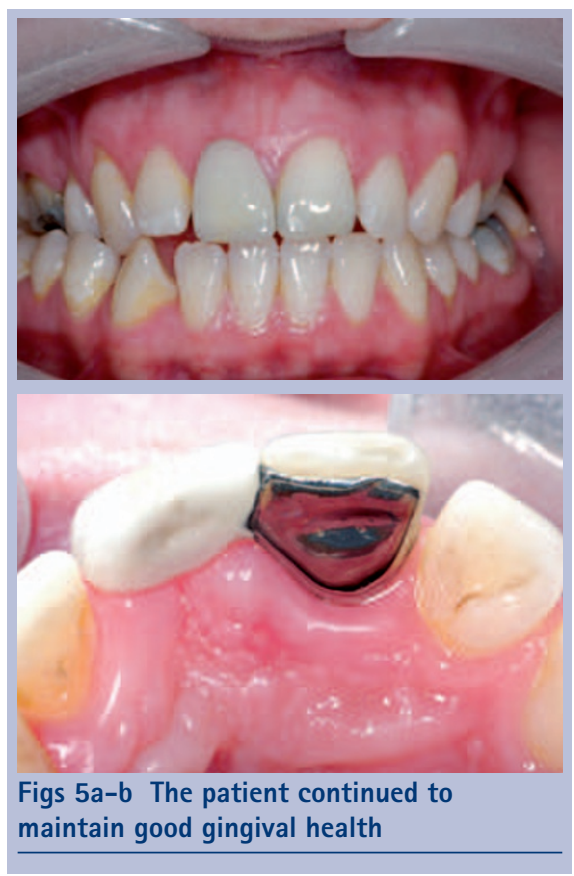

rate for resin bonded bridges to be $87.7 \% .^{8}$ For cases of bridgework, factors such as bridge abutment selection, tooth position, occlusion and parafunctional habits should be assessed and evaluated at the outset before treatment. Patient factors such as compliance and motivation especially with maintaining a disease-free mouth in terms of caries, periodontal health is important. The treatment modality should be realistic and address the patient's concerns as well as adequately fulfil their expectations. Use of resin 'mock ups' in the mouth following a diagnostic wax up is a good method of allowing further discussion and ensuring informed consent.

In this case, the patient's bridge had been re-cemented on two previous occasions. There were excess cement deposits on the palatal and mesial aspects of the abutments, during bridge removal. This is likely to be the result of repeated cementation of the prosthesis which has led to poor bonding and inadequate cement removal. The patient's oral hygiene was also compromised in that region, compounding the problem. This had resulted in gingivitis including hyperplasia of the gingiva, which caused the initially supragingival restoration margin to become subgingival. Overhanging restoration margins or residual excess cement increase the amount of plaque retention and also increases the specific periodontal pathogens in the plaque. ${ }^{1}$

There is a high likelihood that inadequate cement removal (following de-bond and

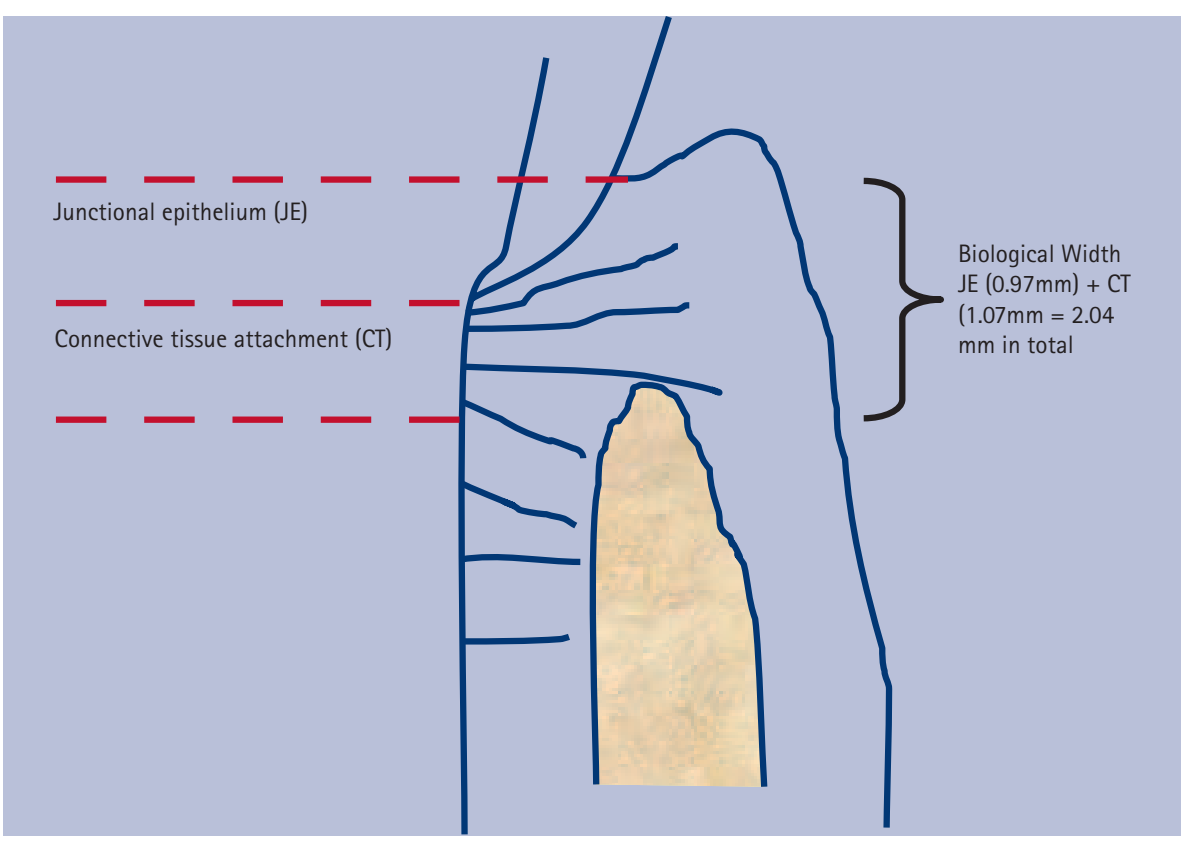

Fig. 6 Biologic width ${ }^{3}$

before re-cementation) at these visits had contributed to the failure of the subsequent cement lute as well as retained plaque at the margins of the retainers. During recementation, the metal retainer should be sandblasted to ensure it is free from any contaminants. Residues of cement should be removed carefully from the abutment surface, without removal of any enamel, so as to allow the restoration to reseat in its original position.

The replacement prosthesis fitted was a cantilever resin-bonded bridge using the 21 as the bridge abutment. Studies have shown that cantilever resin-bonded bridges are more successful than fixedfixed restorations. ${ }^{9,10}$ This bridge design is viewed as more favourable as with two abutments there is more likelihood of failure of the cement lute and subsequent debond due to different degrees of mobility of the teeth. ${ }^{11}$ The main issue with using more than one tooth as an abutment for a resin-bonded bridge is that failures resulting in debond can remain undetected, as the prosthesis continues to remain in situ. If a single bridge abutment is used, the failure is obvious as the whole prosthesis de-bonds and does not remain in situ. Certain clinical situations where the amount of enamel for retention needs to be maximised or where the prosthesis may need to provide an element of periodontal splinting (that is, where previous orthodontic treatment has been carried out) may demand the use of a fixed-fixed design. ${ }^{12}$
The bridge pontic was designed to be of a modified ridge lap design ensuring optimum aesthetics, ease of plaque removal by the patient and causing no trauma to the underlying saddle area. Pontic design is an important factor in preventing mucosal inflammation. Figure 7 shows various pontic designs and ease of cleansibility. The cleansibility of the pontic design is crucial in maintaining the health of the underlying mucosa. The presence of bacterial plaque is essential to the production of mucosal inflammation on the underlying surface of the pontic. ${ }^{16} \mathrm{~A}$ desirable pontic design in conjunction with regular removal of plaque deposits via oral hygiene techniques is necessary to ensure a successful clinical and aesthetic outcome. An ovate pontic design has a convex surface which results in an increased surface area in contact with the adjacent mucosa of the saddle area. ${ }^{17,18}$ The pontic applies a light pressure to the underlying mucosa to achieve an improved aesthetics with the emergence profile. Even though ovate pontics have been used to ensure optimal aesthetics, the close proximity to the mucosa can compromise the ease of cleansibility and therefore there is a greater likelihood of plaque retention and subsequent unwanted sequelae. Hence the need for healthy tissues and effective oral hygiene measures is even greater. In a case such as the one described above, where the pontic site was already inflamed, it would be inadvisable to restore the space using a bridge with a pontic design which is likely to compromise the health of the already 
1. Wash-through pontic

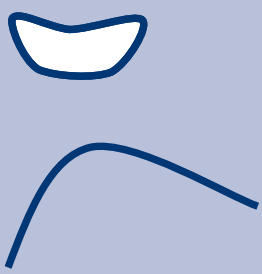

2. Dome pontic

(bullet-shaped or torpedo shaped)

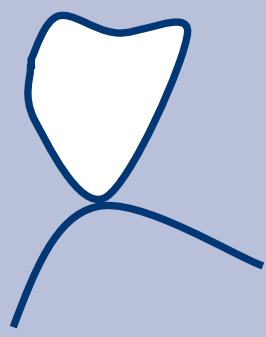

3. Modified ridge-lap pontic

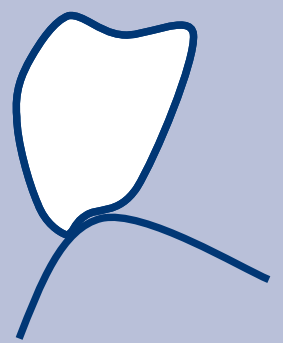

4. Ridge lap pontic (full saddle pontic)

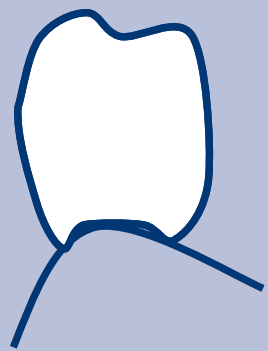

5. Ovate pontic

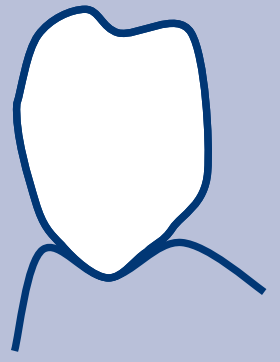

Fig. 7 Types of pontic design (redesigned from references ${ }^{13-15}$ ) inflamed tissues even further. Prior to use of ovate pontics, pontic site development is usually necessary to ensure a concave mucosal surface. Surgical procedures such as electrosurgery can be used. In these instances, use of a removable prosthesis with incremental additions should be considered as well.

Careful attention to clinical technique is required in cases of fixed prosthodontics. Preparation of abutments, good moisture control and contamination of the tooth surface and/or the fitting surface of the retainer are aspects to consider. Recent articles have made recommendations for clinical success of resin bonded bridges. ${ }^{12,19}$

Maintenance of bridgework is usually reliant upon patient compliance with diet advice, and oral hygiene regimens. Periodontal conditions with bridgework are better in individuals who have been implementing oral hygiene measures compared to those patients who have not. ${ }^{20}$ Essential elements for maintaining gingival health are access of the prosthesis for plaque removal and efficacy of the patient's oral hygiene regime. ${ }^{17}$ A research study investigating the effects of oral hygiene procedures on the mucosal and gingival health associated with bridge pontics concluded that even desirable pontic designs do not alone prevent inflammation of the mucosa adjacent to the pontic. ${ }^{16}$ It is the removal of plaque and maintenance of pontic hygiene that allows resolution of chronic inflammation of the adjacent tissues. Clinicians have a duty of care to ensure that patient are adequately educated in how to care for and maintain the bridge prosthesis with demonstrations and advice in using specific oral hygiene aids such as superfloss. Regular visits to the patient's own general dental practitioner will allow opportunity for review and early detection and treatment of failures.

\section{CONCLUSION}

The conservative management in this case achieved the most predictable result for long term success. Although it may be argued that this treatment was lengthy in time, it avoided the need for crown lengthening surgery and gave opportunity for re-stabilisation of the gingivae before provision of a replacement prosthesis. Case selection, design and manufacture of the prosthesis, clinical technique and maintenance are important factors which have a bearing on the successful outcome in such cases. Unfortunately, if these factors are not given due consideration, it can result in a poor aesthetic and functional outcome as well as demand extensive remedial treatment.

1. Padbury A Jr, Eber $R$, Wang $H$ L. Interactions between the gingiva and the margins of restorations. J Clin Periodontol 2003; 30: 379-385.

2. Goodacre C J, Bernal G, Rungcharassaeng K, Kan $J$ Y. Clinical complications in fixed prosthodontics. J Prosthet Dent 2003; 90: 31-41.

3. Gargiulo A, Wentz F, Orban B. Dimensions and relations of dentogingival junction in humans. J Periodontol 1961: 32: 261-267.

4. Rosenburg E S, Garber D A, Evian C I. Tooth lengthening procedures. Compend Contin Educ Gen Dent 1980; 1: 161-172

5. Wagenberg B D, Eskow R N, Langer B. Exposing adequate tooth structure for restorative dentistry. Int J Periodontics Restorative Dent 1989; 9: 322-333.

6. Hussey D L, Linden $\mathrm{G} J$. The clinical performance of cantilevered resin-bonded bridgework. J Dent 1996; 24: 251-256.

7. Rashid S, Abidi Y A, Hosein T. Success rates of resin-bonded restorative dentistry bridges. J Coll Physicians Surgic Pak 2003; 13: 684-687.

8. Pjetursson B E, Tan W C, Tan K, Bragger U, Zwahlen $M$, Lang N P. A systematic review of the survival and complication rates of resin-bonded bridges after an observation period of at least 5 years. Clin Oral Implants Res 2008; 19: 131-141.

9. Olin PS, Hill E M, Donahue J L. Clinical evaluation of resin-bonded bridges: a retrospective study. Quintessence Int 1991; 22: 873-877.

10. Kern M. Clinical long-term survival of two-retainer and single-retainer all-ceramic resin-bonded fixed partial dentures. Quintessence Int 2005; 36: 141-147.

11. Shillingburg $H T$, Hobo $S$, Whitsett $L D$, Jacobi $R$, Brackett S E. Fundamentals of fixed prosthodontics, 4th ed. USA: Quintessence Publishing Co. Inc., 2011.

12. Barber $M$ W, Preston $A J$ J. An update on resinbonded bridges. Eur J Prosthodont Restor Dent 2008; 16: 2-9.

13. Becker C M, Kaldahl W B. Current theories of crown contour, margin placement and pontic design. J Prosthet Dent 1981; 45: 268-277.

14. Smith B G N, Howe L C. Planning and making crowns and bridges. 4th ed. Oxon: Informa Healthcare, 2006.

15. Tay W M. Resin bonded bridges: a practitioner's guide. London: Martin Dunitz Ltd., 1992.

16. Silness J, Gustavsen F, Mangersnes K. The relationship between pontic hygiene and mucosal inflammation in fixed bridge recipients. J Periodontal Res 1982: 17: 434-439.

17. Gahan M J, Nixon P J, Robinson S, Chan M F. The ovate pontic for fixed bridgework. Dent Update 2012; 39: 407-415.

18. Abrams L. Augmentation of the deformed residual edentulous ridge for fixed prosthesis. Compend Contin Educ Gen Dent 1980; 1: 205-213.

19. Durey K, Nixon PJ, Robinson S, Chan M F WY. Resin bonded bridges: techniques for success. Br Dent J 2011; 211: 113-118.

20. Silness J. Periodontal conditions in patients treated with dental bridges. J Periodontal Res 1970; 5: 60-68. 